\title{
Improved Disk-Drive Failure Warnings
}

\author{
Gordon F. Hughes, Fellow, IEEE, Joseph F. Murray, Kenneth Kreutz-Delgado, Senior Member, IEEE, and \\ Charles Elkan
}

\begin{abstract}
Improved methods are proposed for disk-drive failure prediction. The SMART (Self Monitoring and Reporting Technology) failure prediction system is currently implemented in disk-drives. Its purpose is to predict the near-term failure of an individual hard disk-drive, and issue a backup warning to prevent data loss. Two experimental tests of SMART show only moderate accuracy at low false-alarm rates. (A rate of $0.2 \%$ of total drives per year implies that $20 \%$ of drive returns would be good drives, relative to $\approx 1 \%$ annual failure rate of drives). This requirement for very low false-alarm rates is well known in medical diagnostic tests for rare diseases, and methodology used there suggests ways to improve SMART.

Two improved SMART algorithms are proposed. They use the SMART internal drive attribute measurements in present drives. The present warning-algorithm based on maximum error thresholds is replaced by distribution-free statistical hypothesis tests. These improved algorithms are computationally simple enough to be implemented in drive microprocessor firmware code. They require only integer sort operations to put several hundred attribute values in rank order. Some tens of these ranks are added up and the SMART warning is issued if the sum exceeds a prestored limit.

These new algorithms were tested on 3744 drives of 2 models. They gave 3-4 times higher correct prediction accuracy than error thresholds on will-fail drives, at $0.2 \%$ false-alarm rate. The highest accuracies achievable are modest $(40 \%-60 \%)$. Care was taken to test will-fail drive prediction accuracy on data independent of the algorithm design data.

Additional work is needed to verify and apply these algorithms in actual drive design. They can also be useful in drive failure analysis engineering. It might be possible to screen drives in manufacturing using SMART attributes. Marginal drives might be detected before substantial final test time is invested in them, thereby decreasing manufacturing cost, and possibly decreasing overall field failure rates.
\end{abstract}

Index Terms-Disk drive, failure prediction, magnetic recording, predictive failure analysis, SMART.

\section{ACRONYMS $^{1}$}

ATA standard drive interface, desktop computers

CSS contact start-stops

DCL DC loop bias level on chip

Manuscript received July 10, 2000; revised June 22, 2001. This work was supported by the UCSD Information Storage Industry Center, funded by the Alfred P. Sloan Foundation. Responsible Editor: F.-B. Sun.

G. F. Hughes and J. F. Murray are with the Center for Magnetic Recording Research, University of California, San Diego, La Jolla, CA 92093 USA (e-mail: GFHughes@ucsd.edu; JFMurray@ucsd.edu).

K. Kreutz-Delgado is with the Department of Electrical and Computer Engineering, University of California, San Diego, La Jolla, CA 92093 USA (e-mail: Kreutz@ece.ucsd.edu).

C. Elkan is with the Department of Computer Science, University of California, San Diego, La Jolla, CA 92093 USA (e-mail: Elkan@cs.ucsd.edu).

Publisher Item Identifier 10.1109/TR.2002.802886.

\footnotetext{
${ }^{1}$ The singular and plural of an acronym are always spelled the same.
}

FA failure analysis of apparently failed drive

FAR false-alarm rate, 100 times probability value

FLY fly height, head/disk

GDC grown defect count

GMX maximum G's mechanical shock

MSE mean square-error in data read detector

MVRS multivariate rank-sum statistical test

NPF drive failed, but No problem found in FA

$\mathrm{POH} \quad$ power-on hours of drive

RAID redundant array of independent disks

RRT recalibrate retry in track seek servo

RSE read soft error in data detector

$s$ - implies the statistical meaning

SCSI standard drive interface, high-end computers

SKE seek errors in track servo

SUT spinup time, to drive ready

SMART self monitoring and reporting technology

TAS thermal asperity count

TMP internal-drive temperature

TMR Offtrack error in track servo

WA failure warning accuracy (probability).

\section{NOTATION}

$n \quad$ number of reference (old) measurements

$m \quad$ number of warning (new) measurements

$N \quad(n+m)$ : total ranked measurements

$p \quad$ number of different attributes measured

$Q(X) \quad$ Gaussian probability $\operatorname{Pr}\{x>X\}$

RS rank-sum statistical hypothesis test

$R \quad$ sum of ranks of warning set data

$R_{c} \quad$ critical value of $R$ : predict fail if $R>R_{c}$

$R_{i} \quad$ rank-sum of attribute $i$ considered alone

$\mu \quad$ mean

$\sigma \quad$ standard deviation.

\section{INTRODUCTION}

$\mathbf{C}$ OMPUTER disk drives are reliable data storage devices with annual failure rates of $0.3 \%$ to $3 \%$ per year [1], [2]. (A $1 \%$ nominal failure rate is used for comparisons in this paper.) Nonetheless, drive failure can cause a catastrophic loss of user data. This is often far more serious than the hardware cost of replacing the failed drive. If impending drive failure could be predicted, then a warning could be issued to the drive user to back up the data onto another storage device.

In 1995, the drive industry adopted SMART: a standardized specification for such failure warnings. SMART is based on monitoring a number of internal drive technology measurements relevant to impending failure. A failure warning algorithm is run 
by the drive microprocessor firmware. This checks whether the measurements exceed maximum thresholds and produces a binary (won't-fail/will-fail) warning. The SMART warning-time goal is 24 hours before drive failure.

Computer operating systems can issue standardized drive commands to enable and to read this failure warning. These commands are defined for the 2 predominant computer-to-drive interface standards, ATA and SCSI [3], [19]. Additionally, the SCSI Enclosure Services specification allows RAID array controllers to be notified if thresholds are exceeded on drive external environments such as power supply voltage, current, and ambient temperature [3], [19].

SMART technology is implemented in most 3.5-inch disk drives manufactured today, the most widely used disk-drives from personal computers to supercomputers. However, it is unknown how many computer systems today enable or read the SMART warning. In some personal computers, SMART is checked on computer bootup by the CMOS/BIOS firmware. Drive manufacturers supply diagnostic programs that read the SMART warning. Information on SMART warning accuracy is anecdotal at best, and the drive internal monitoring technology is manufacturer proprietary.

This paper assesses the accuracy of the existing SMART failure-warning algorithm in drives, and an improved algorithm. Experimental data are from drive-design reliability testing of 2 different Quantum Corporation disk-drive models. Trade-off curves of the WA are calculated versus the FAR (defined as the probability that a fail warning will occur in a drive that does not subsequently fail).

\section{FAILURE WARning TeChNOLOGY}

\section{A. Background}

Failure warning markedly differs from usual disk-drive reliability methodology. The latter statistically predicts failure probability over an entire drive population, and assumes that all drives are equally likely to fail [2], [4], [5]. SMART predicts individual drive failure.

Failure warning technologies such as condition-monitoring and predictive-maintenance are also used, in process control and large motor monitoring [6]-[8].

\section{B. SMART Disk-Drive Failure Warning}

The SMART ATA drive specification [3], [19] allows up to 30 internal drive measurements. These are termed failure attributes and are periodically measured by a drive. Attribute values are stored in the "drive reserved data area" with other drive operational parameters. For a drive user to receive a SMART warning, the computer system must issue specific drive interface commands to enable the algorithm and then to read the resultant won't-fail/will-fail warning [9]. Some drives will unilaterally shut down if internal sensors detect extreme temperature or mechanical g-shock [10].

Maximum thresholds are defined for each attribute by the drive manufacturer. The SMART warning-flag is set in response to an ATA SMART Return Status command, if any attribute exceeds its threshold. This is a logical OR operation among the several attribute threshold tests, and is used
TABLE I

SMART ATTRIBUTES

\begin{tabular}{c|l|l} 
Symbol & Relevance & Key \\
\hline POH & Drive aging & C \\
CSS & Drive power cycles & C, F \\
SKE & Heads seek to wrong track & I,T \\
RRT & Drive re-initializes & I \\
RSE & Errors corrected by inner ECC code & I, F, T \\
MSE & Precursor of RSE & I \\
GDC & New disk defects, found after manufacture & C, F, T \\
SUT & Power on to drive ready & I, F \\
TMR & Head-track misregistry & I,T \\
GMX & Mechanical shock & I \\
TAS & Thermal asperity count & I,F \\
FLY & By PW50, or Wallace, or read IC MSE, & \\
& FIR taps & I \\
TMP & Drive max temperature limit & I \\
DCL & Read IC ok & I \\
- & Spin \& Track Motors: Acoustics, & \\
& start current, or control loop analysis & I,T \\
\hline
\end{tabular}

Keys: C (cumulative measurement), I (incremental),

$\mathrm{F}$ (head/disk interface indicative), $\mathrm{T}$ (track servo indicative)

because some drive failures can be predicted by only one attribute. But this OR operation can also cause a high false-alarm rate, because it does not require multiple confirming attribute signatures to trigger the warning.

Table I lists SMART attributes, starting with basic nearly universal attributes, to proposed future attributes. The basic attributes exploit existing drive internal technology (thus allowing minimal added cost). Many were historically adopted for drive error recovery and for reliability analysis, with SMART warning thresholds added later. Most attributes are increment al error counts over a fixed time interval. For example, certain rates of seek and read soft errors are allowed by drive designers, and if the incremental counts of these errors remains stable, then failure is not indicated. Cumulative counting would mislead.

$\mathrm{POH}$ is a traditional measure of drive age. Low POH can imply infant-mortality failure risk and high $\mathrm{POH}$ can imply end-of-life failure risk. But for failure warning, both need corroboration by other attributes. A related attribute is CSS, which is a count of drive power cycles; i.e., power on, disks spin up, heads fly, power off, heads contact disk while spinning to stop. High CSS increases the risk of head/disk sliding contact wear. (Some drives avoid head-disk contact and have no CSS attribute). These attributes are cumulative.

SKE is an incremental count of track seeks that require a second re-seek to find the intended track. The count is reset to 0 after a fixed number of thousands of seek commands. If a re-seek also fails, then a RRT reinitializes the head tracking servo system, and is counted in a separate RRT attribute.

An RSE is a data read error detected and corrected by an error correction code. It can indicate disk defects, high head fly, or head off-track. Repeated RSE errors at the same user data disk location can invoke drive error recovery which moves the user data to a new location, and records a GDC. Read channel parameters such as the Viterbi detector MSE can warn of an approaching GDC before an RSE occurs [11].

SUT is the elapsed time from power-on to drive "ready for data transfer." Increasing SUT can indicate head-disk stiction (static friction), raising the risk that the drive spin motor might 
be close to its maximum starting torque limit. Disk spin motor current and spin servo parameters can detect late head fly takeoff, bearing damage, or runout.

TMR monitors the track servo misregistry error signal [12], which can indicate mechanical G-shock, head mechanical resonance faults, or spindle bearing runout. It is also used to inhibit writing, to eliminate the risk of corrupting data on an adjacent track.

FLY can be measured using magnetic recording physics, such as playback pulse width PW50, or the pulse peak amplitude normalized by pulse area, or read channel equalization parameters [11]. The Wallace spacing loss formula can also be used [13]. One head flying appreciably high (compared to the average of other heads in the drive) indicates a risk of poor writing or reading, and a low fly head increases head-disk wear risk.

TMP is measured by some manufacturers using a dedicated thermal sensor in the drive, or from read preamplifier outputs which indicate the magneto-resistive read sensor resistance, and hence its temperature. High temperature stresses the electromechanics of the drive, and low temperature can allow moisture condensation on the disks, leading to head stiction. G-shock can be monitored (GMX) by a G-sensor.

\section{The Failure-Rarity Problem}

SMART failure-warning algorithms can be tested using experimental data sets of periodic attribute reads over the life of drives. Times when drives appear to fail are noted, and failure is verified by physical failure analysis. Because drive failure rates are only about $1 \% / y e a r$, thousands of drives must be tested for more than a year to get $s$-significant numbers $(>15)$ of failed drives. This is larger than the number of drives in most large RAID arrays, even in many supercomputers.

Controlling the false-alarm rate places the most critical demand on SMART warning algorithms. A seemingly small falsealarm rate of $1 \% / y e a r$ would double the total number of drives returned for failure, because this rate is about equal to actual annual failure rates. This requirement for very low false-alarm rates is well-known in medical diagnostic epidemiology tests for rare diseases [14].

One good source of experimental data (used here) is testing new drive designs. Typically, several thousand drives of a new design are tested by a drive manufacturer to expose latent design and reliability problems. Thus, more failures are anticipated than in production drives. The testing includes drives with experimental components or built under experimental conditions that are not used in full-scale mass production. Consequently, appreciable numbers and types of failures are likely to occur. This has the advantage of producing more failures for statistical SMART test development. However, caution is necessary to guard against failure modes caused by test conditions rather than inherent drive technology. The test data used here seem to be valid for SMART analysis because the failure types are representative and typical of field failures.

FA is performed to verify failed drives and determine failure causes for corrective redesign. Typically $20 \%-30 \%$ of apparently failed drives are NPF drives, which operate normally when analyzed. Therefore, FA is important for a valid data set, and is also highly effective in gathering definitive failure data and statistics, which can guide attribute performance and selection.

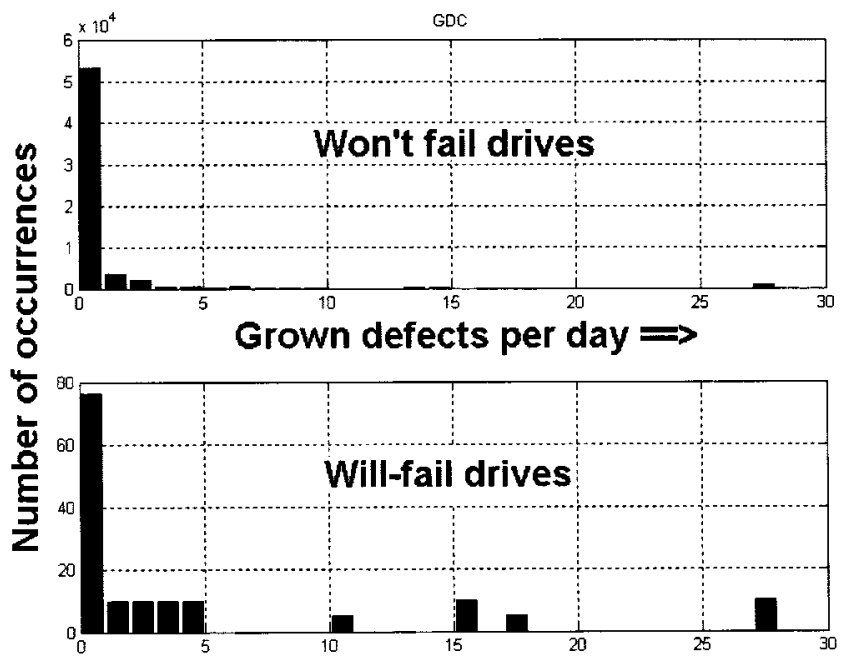

Fig. 1. Grown-defect data histograms: All won't-fail drives (top) versus all will-fail drives (bottom).

These NPF rates also imply that disk-drives have a false-alarm rate of $0.2 \%-0.3 \%$ even in the absence of SMART (20\%-30\% of the $1 \%$ year perceived failure rate).

Another possibility (not tried here) is to mathematically-characterize the experimental data sets in order to generate simulated attribute data using Monte Carlo methods.

\section{Attribute-Data Characteristics}

In addition to the SMART warning flag, the original ATA SMART specifications [3], [19] define a 512-byte SMART datarecord format. This allows the drive internal SMART attributes to be read out, as 1-12 byte integers (raw, unnormalized attribute data are used here [3], [19]).

Figs. 1-3 shows histograms of GDC, SKE, and RSE attribute data from 1 of the 2 drive design tests. Data on top are from "won't-fail drives," and data below are from "will-fail drives" (which subsequently failed during the test). These histograms are all the attribute data from all the drives of one model, to illustrate the nature of the attribute data. For example, there were about 53000 occurrences of 0 grown defects among all the attribute data reads taken from all drives that did not fail, and 75 0 -GDC reads from drives that did ultimately fail during the test.

The characteristic distinguishing the "will-fail drives" from the "won't-fail drives" is a pattern of high attribute values during a warning measurement interval. A single high value could be a statistical or transitory accident, but a scatter dominance pattern appears important in Figs. 1-3. Precise attribute values are not as important as a pattern of scatter.

In statistical terms, this is ordinal data, in that increasing attribute values imply increased failure risk, but a doubled attribute value does not necessarily double the risk.

Although there appears to be randomness in these histograms, they do not resemble continuous parametric distributions, such as Gaussian or Weibull. Because drive failure is caused by important physical changes, the only relationship between will-fail and won't-fail drive data can be simply that they appreciably differ from each other, more than would be anticipated from statistical noise. 


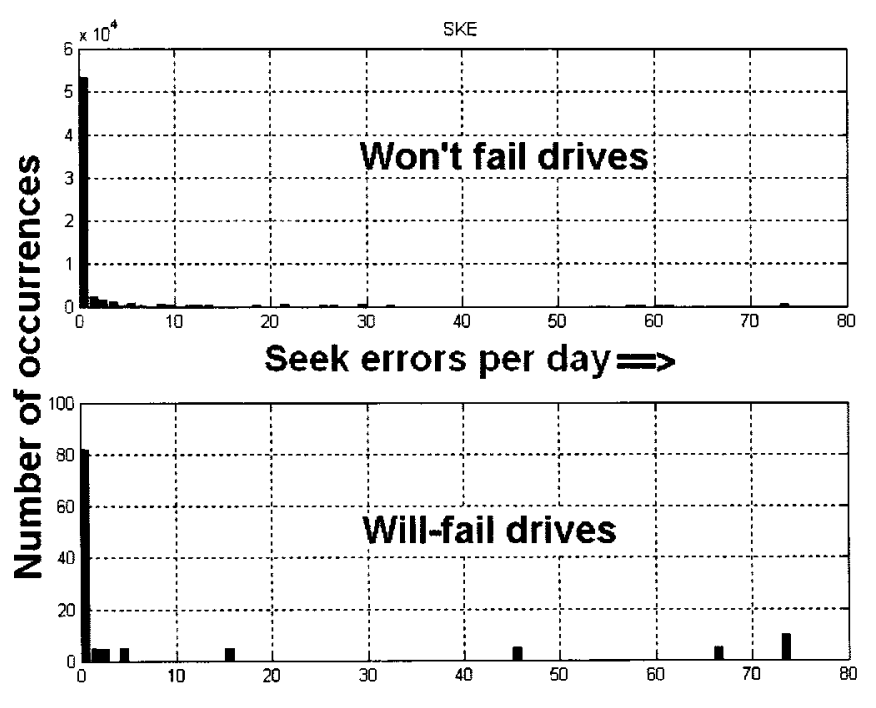

Fig. 2. Seek errors histograms: All won't-fail drives (top) versus all will-fail drives (bottom).

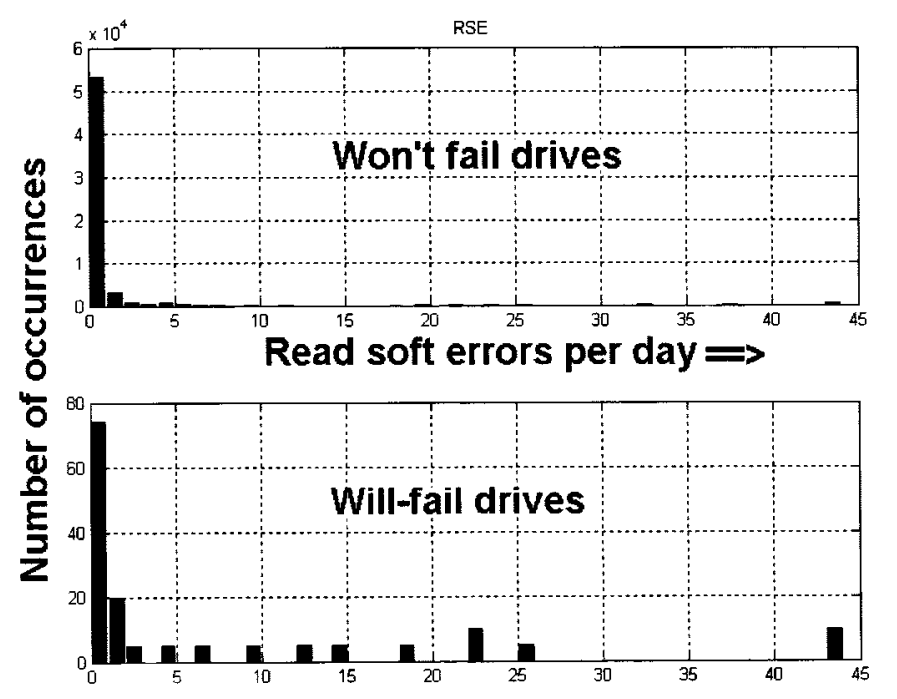

Fig. 3. Read soft errors histograms: All won't-fail drives (top) versus all will-fail drives (bottom).

\section{E. Special Data Factors in Drive Design Tests}

Low POH and CSS values generally indicate infant mortality risk; and high values suggest increased end-of-life failure potential. But these are not conclusive without other confirming attributes. Additionally, in drive design tests of fixed duration, these attributes can appear $100 \%$ predictive but misleading. (All won't-fail drives get the full test duration POH and CSS; will-fail drives obviously get less.)

Drive design testing is done to expose latent problems, in order to eliminate them by redesign. The drives being tested have higher failure rate than mass production drives (see Section II-C). This is similar to medical research conditions that accelerate the disease under study [14].

Using the same drive test data, both to select a SMART algorithm and to test its accuracy, can be misleading. Here, the algorithm parameters are selected to give an acceptable FAR on won't-fail drives, and then tested for WA on a different data set, viz., the will-fail drives. In addition the same algorithm is tested on two independent drive models. The FAR on production drive data should be lower, because the test purpose is to remove drive design failure modes.

\section{F. Rank-Sum Statistical SMART Tests}

SMART algorithms can be regarded as statistical hypothesis tests. They use SMART data to test the hypothesis that "a drive will fail" against the null hypothesis that "a drive is remaining stable and will not fail." The existing SMART threshold algorithm uses only the most recent attribute values, and issues a failure warning if any attribute is above its critical failure threshold. This is a logical OR of independent tests on each attribute.

Wilcoxon rank-sum [15] statistical tests are proposed here to replace the threshold tests, to improve failure warning accuracy and to lower false-alarm rates.

Rank-sum tests are widely recommended for rare failure situations (such as rare disease epidemiology) where false-alarms are costly. They are particularly useful when the statistical distributions are unknown and suspected of being non-Gaussian [14], [16].

For drive failure warning, an appropriate hypothesis test is to use a warning data set of recent attribute values and then to compare it to an original reference data set taken from the drive population during manufacture. If the 2 data sets vary only in probable statistical noise, then the null hypothesis is selected. Namely, the drive is stable and no warning is issued. Figs. 1-3 illustrate the general idea: the upper histogram of each attribute represents the reference set; the lower histogram represents the warning set. (But the warning histogram would be data from an individual drive, not all the drives as in Figs. 1-3.)

The warning data set for each drive is taken to be its latest 5 samples of each attribute (the most-recent 5 days of data for these drive models).

The reference data set for each attribute is taken to be 50 random samples of that attribute taken from initial SMART reads; and averaged over many good drives. The optimum data set sizes depend on factors such as the SMART attribute read interval. These data set sizes gave the best WA and FAR results for these test data.

The best reference sets were using the first few attribute values from the (several thousand) good drives. They were randomly divided into 50 groups, and each attribute for the group taken as the (single) average of 50 values (rounded to an integer). Will-fail drives are not included in the reference-set averaging. They can be kept as $s$-independent data for predictive accuracy testing. (Even if they were included in a production-drive situation, $a \approx 1 \%$ FAR implies that the averaging should wash out their influence.)

Thus, if 50 typical measurements from new drives have 1 or 2 SKE, an example SKE reference data set might consist of 48 "0"s, a single " 1 " count, and a "2" count (in any order).

The warning and reference data sets might look like Fig. 3, with many ties at the lowest rank (zero seek errors), and the maximum rank being 1 instance of 43 seek errors in this 1-day time interval. (Fig. 3 is actually all drives.)

The rank-sum test for a given drive is computed numerically by a sorting operation on the combined warning and reference data sets for each attribute. The reference data never change for 
a given drive, but its warning data do change because it is the last 5 samples before the SMART warning test is to be made.

Rank-1 is given to the smallest combined attribute value, rank-2 to the next, et al. The rank-sum statistic is the sum of the ranks of the warning attribute values, among all the attribute values in the warning and reference data sets. If the drive is stable, the warning data ranks should intersperse randomly among the reference data ranks, because the 2 data sets have the same statistical distribution (which does not have to be known). If the rank-sum is higher than a fixed limit (precalculated and stored in the drive), the test concludes that the 2 data sets have distinct statistical differences, within a specified FAR. This implies that the drive attributes have statistically changed since manufacture, indicating potential failure.

\section{G. Numeric Example of Rank-Sum Test}

The Appendix presents the mathematics and derivation of the rank-sum test. The following numeric example demonstrates it.

Consider the made-up seek error data in Table II. Each reference datum in column 2 is a seek error count over a specified SMART frame interval, randomly taken from good drives. There are 8 occurrences of 1 seek error, 3 occurrences of 2 errors, and 1 occurrence of 4 errors. These reference data never change.

The latest warning data error counts from 1 drive are in column 3 . They show a pattern of higher counts, qualitatively suggesting that this drive is now making more seek errors and might fail.

Column 4 shows the total ranks of columns 2 and 3 data. When ties occur at any error count, then all the tied data are given the average rank of the ties. (Appendix A.4 discusses why data ties are given their average rank, and why 0 error counts are ignored.) The rank for the $8+1=9$ error counts of 1 seek error is therefore the average rank of 1 and 9 for all 9 error counts. Thus, the rank of the single seek error count of 1 in the warning set is $(1+9) / 2=5$. This is the first term in the warning set rank-sum, shown in column 5 . There are $3+2=5$ error counts of 2, taking total ranks 10-14, 2 of which are in the warning data; thus the rank-sum gets 2 entries with the average rank of 12. There is only 1 warning error count of 3 ; thus the warning rank-sum gets its rank of 15 . The next warning rank is at 5 errors, and its total rank of 17 goes into the rank-sum. There are no 6-error counts, and the rank-sum gets the next rank of 18 for its single 7-error count. The resulting rank-sum is 79. No error count datum is ignored-the rank averaging of the ties keeps the total rank count equal to the total $12+6=18$ error counts.

If the 18 total rank numbers $s$-independently result from the same probability distribution, then the rank-sum of the 6 warning data should be the sum of 6 random integers from the set $1,2, \ldots, 18$. Each datum has an equally random rank if all are $s$-independently drawn from any single distribution. The average rank-sum should be about 6 times the average integer: $6 \cdot(1+18) / 2=57$. The rank-sum variance should be 6 times the variance of a uniform probability distribution with range from 1 to 18 ; thus

$$
\sigma=(18-1) \cdot \sqrt{\frac{6}{12}}=12.0 \text {. }
$$

The warning rank-sum of 79 is $(79-57) / 12=1.8 \sigma$ above its mean, appreciably higher than from random probability. (These rough statistics are from Appendix A.1.)
TABLE II

EXAMPLE RANK-SUM SEEK ERROR DATA

\begin{tabular}{c|cccl}
$\begin{array}{c}\text { Seek } \\
\text { Errors }\end{array}$ & $\begin{array}{c}\text { Reference } \\
\text { data }\end{array}$ & $\begin{array}{c}\text { Warning } \\
\text { data }\end{array}$ & $\begin{array}{c}\text { Total } \\
\text { Ranks }\end{array}$ & $\begin{array}{l}\text { Warning } \\
\text { Ranks }\end{array}$ \\
\hline 1 & 8 & 1 & $1-9$ & $(1+9) / 2=5$ \\
2 & 3 & 2 & $10-14$ & 12,12 \\
3 & 0 & 1 & 15 & 15 \\
4 & 1 & 0 & 16 & - \\
5 & 0 & 1 & 17 & 17 \\
6 & 0 & 0 & - & - \\
7 & 0 & 1 & 18 & 18 \\
Sums & 12 & 6 & & 79 \\
\hline
\end{tabular}

This rank-sum procedure is repeated for each drive attribute. The results are combined into a single drive failure warning, if any rank-sum exceeds a maximum threshold (as in present diskdrive SMART), or if a single overall rank-sum can be computed (see Section II-B).

\section{H. Rank-Sum Test Advantages}

Several advantages follow from rank hypothesis testing.

1) The rank warning is based on a $s$-significant test that a warning data set differs from the reference set, instead of the single data point used in threshold SMART. This can lower the FAR by statistical averaging.

2) The rank-sum test makes no mathematical assumptions nor needs any information about the statistical distribution function of the data. It assumes only that: a) the data have some fixed distribution if the drive is remaining stable, and b) the attribute samples are $s$-independent.

3) Rank-sum is a stochastic-dominance test based on ordinal statistics. This means that failure risk is increasing if the attribute values are statistically increasing, but no numerical proportionality is assumed.

4) The ranks are relatively immune to errors in the attribute data. Extreme value outliers merely get the maximum rank no matter how large they are.

5) Summing the ranks exploits the known monotonicity of the attributes - attributes are defined such that larger values imply increased failure risk (see Section II-D).

6) Rank-sum mathematics is simple enough to implement in disk-drive firmware, requiring only sorting and adding of attribute integer values. The Appendix presents the mathematics of the rank-sum test.

\section{Multivariate Tests versus ORing Single Attribute Tests}

Like the threshold SMART algorithm, rank-sum SMART tests attributes individually, and issues the SMART warning if any attribute is appreciably increasing. Combining single-attribute hypothesis tests by this OR operation (also used in threshold SMART) could increase the false-alarm rate.

A warning algorithm based on the entire set of warning and reference attribute data could offer higher predictive accuracy at lower false-alarm rate by exploiting statistical correlations between the attributes. A multivariate SMART decision rule was developed for this purpose (Appendix A.4). It can operate on variables defined so that increasing-values imply increasing failure-risk. This covers all attributes in Table I, except for possibly using POH/CSS to capture infant-mortality failures. (For 
TABLE III

DRIVE TEST SUMMARY

\begin{tabular}{l|rr} 
Drive Test ID: & A & B \\
\hline Drives in test & 1936 & 1808 \\
Fail drives & 9 & 27 \\
Fail analysis but NPF & 6 & 11 \\
Attribute reads: & 94071 & 63153 \\
Significant attributes: & GDC, SKE, RSE & GDC, SKE, RSE \\
\hline
\end{tabular}

that situation, the highest rank could be put on the smallest $\mathrm{POH} / \mathrm{CSS}$ values, but this simple inversion would be unable to test for end-of-life failure risk.)

\section{EXPERIMENTAL RESULTS}

Experimental data sets were obtained from drive design testing of 3744 drives of 2 different Quantum Corporation drive models (Table III). Each set contains 2-3 months of reliability design test data. There were 36 verified drive failures $(1.0 \%$, or $4 \%-6 \%$ annual rate, see Section II-C). The attributes found most predictive in these data were: GDC, SKE, RSE. These 3 attributes are also physically reasonable for the actual drive-failure causes. Examples of verified failure causes in model $A$ drives and their SMART attribute warnings are:

- grown defects from disk mechanical misalignment (GDC warning);

- mobile thermal asperities (foreign particles on the disk) causing GDC;

- unstable servo due to head problem (SKE);

- head arm flex cable electrically intermittent (RSE);

- head instability (RSE);

- head burst noise (RSE).

These are typical design failure types with perhaps an unusually large number of head problems.

Figs. 4 and 5 show tradeoff curves of WA versus FAR, for the 2 drive models A and B. In Figs. 4 and 5, the NPF drives are grouped with the won't-fail drives. But because the NPF drives did apparently suffer some transient failure during the test, internal damage might have occurred even though failure analysis found them operational and they were returned to the testing. Such NPF failures are also often caused by external problems like drive power supply, connectors, and ambient temperature. The dotted line shows the change in the multivariate rank-sum test, if NPF drives are grouped with the will-fail drives. Calling the NPF drives failures, lowers the rank-sum accuracy from about $32 \%$ to $20 \%$ at $0.2 \%$ FAR.

Fig. 4 shows an ORed rank-sum test correct warning probability of about $40 \%$, at $0.2 \%$ FAR probability. The multivariate rank-sum is similar. Conventional SMART ORed thresholds have warning accuracy 3-4 times lower, at $0.2 \%$ FAR.

Fig. 5 shows overall poorer results for drive model $B$. Multivariate rank-sum is best at $12 \%$ with ORed rank-sum and ORed threshold tests at $4 \%$, both at $0.2 \%$ FAR. However, this data set was difficult to analyze: 14 of the 1808 nonfail $B$ drives had corrupted data. These were eliminated. Of the 27 will-fail $B$ drives, 18 had all-zero attribute reads and their failure is unpredictable by any method. (If these 18 drives are ignored, the $12 \%$ WA increases to $36 \%$.) Additionally, only 57 of the 1724 won't-fail

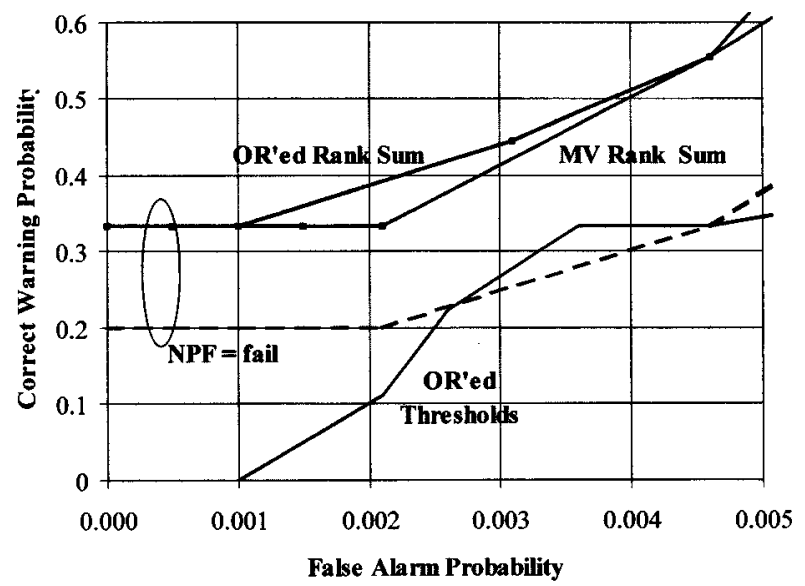

Fig. 4. Drive model $A$ : Warning accuracy versus false-alarm rate. Dotted curve: MV rank-sum if NPF drives are called fails.

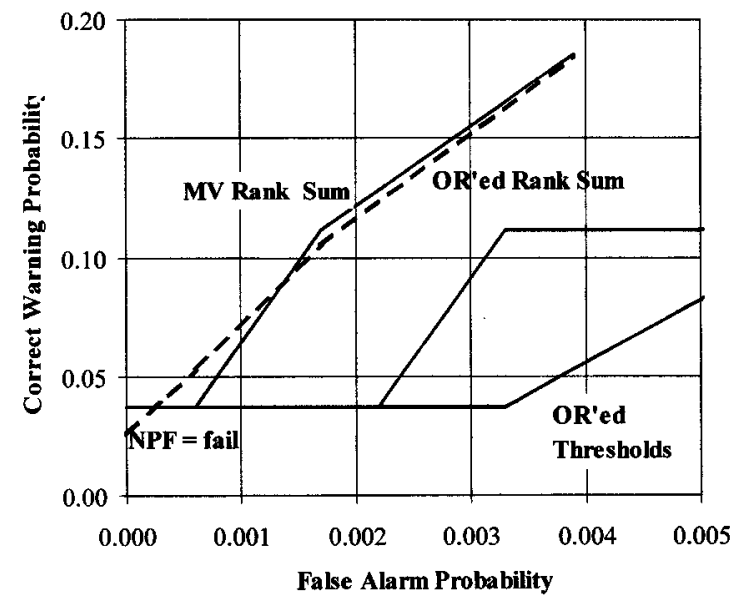

Fig. 5. Drive model $B$ : Warning accuracy versus false-alarm. Dotted curve: MV rank-sum if NPF drives are called fails.

drives had any nonzero attributes. This weakens the reliability of the FAR values obtained on $B$.

If SMART warnings were used to signal drive replacement, the $0.2 \%$ FAR quoted above might be acceptable by drive manufacturers. It would put a $20 \%$ limit on the increase in apparently failing drives, compared to a nominal $1.0 \%$ annual drive failure rate. It is also roughly the false-alarm rate with no SMART at all (20\% NPF drives in 1\% perceived-failure drives per year). For a drive user, this FAR might be acceptable if the $40 \%$ WA in Fig. 4 were a useful accuracy. In a RAID array or enterprise storage network, higher FAR might be acceptable, if SMART is used to trigger data backup instead of drive replacement. The highest WA attainable in $A$ drives was $60 \%$, at $0.5 \%$ FAR.

These results indicate appreciable accuracy improvement potential over present SMART, tested on two $s$-independent drive models, using the same new SMART algorithms. However, results from drive-design test-data do not prove anticipated WA in production drives.

Additionally, disk-drives have a variety of possible failure modes; not all monitored by SMART attributes; thus, the WA cannot be $100 \%$. Two of the 9 model $A$ drive fails were unpredictable because their attribute-reads were all 0s. Figs. 4 and 5 may show the highest realistic WA with the present attributes. 


\section{APPENDIX}

\section{A.1 Rank-Sum Hypothesis Test}

Consider an individual attribute, $x$, a set of $m$ warning measurements, $x_{k}$, and a reference data set of $n$ measurements. For example, the warning set in any individual drive being the last $m=5$ read soft error counts per SMART read interval of (say) 8 or 24 hours, stored as few-byte integers. The reference data set might be $n=50$ reads taken from nominal drives that passed design testing. All drives of one model made in one production configuration could have the same reference-set data stored in them.

The rank-sum algorithm first puts all $N=n+m$ attribute measurements in rank order, ignoring which data set they came from, with the highest rank on the numerically largest measurement. This is a simple integer sort. Then the numerical ranks of the $m$ warning set measurements are added up. The resultant rank-sum $R$ is compared to a precomputed limit $R_{c}$ (2-byte rank-summing and a 2-byte critical limit constant stored in the drive firmware code is sufficient for $N=250$ data samples). $R_{c}$ is computed under the null hypothesis that both data sets are from the same distribution, using its mean $\mu$ and variance $\sigma^{2}$ [17], [18]:

$$
\begin{aligned}
\mu[R] & =\frac{1}{2} m \cdot(N+1), \\
\sigma^{2}[R] & =\frac{1}{12} n \cdot(N+1) \cdot m, \\
\frac{\sigma}{\mu} & =\sqrt{\frac{n}{3 m \cdot(N+1)}} .
\end{aligned}
$$

Using $\mu$ and $\sigma$, the $s$-significance level (false-alarm probability) of the rank-sum test is used to calculate a critical rank-sum value limit $R_{c}$, using the single-tail Gaussian distribution $Q(X)=$ $\operatorname{Pr}\{x>X\}$, with $X \equiv\left(R_{c}-\mu\right) / \sigma$ :

$$
\mathrm{FAR}=Q\left[\frac{R_{c}-\mu}{\sigma}\right] .
$$

If a warning test, $R$, exceeds $R_{c}$, then the 2 data sets are statistically dissimilar and failure should be predicted.

This Gaussian distribution approximation is widely used if $n, m>20$, but is inadequate with this SMART data set, and $R_{c}$ was numerically set (Appendix A.6) to get a desired FAR, producing Figs. 4 and 5 .

\section{A.2 Simplified Rank-Sum Mathematical Derivation}

Under the null hypothesis (drive is remaining stable), all $N=$ $n+m$ measurements are $s$-independent samples from the same statistical distribution. This distribution can be discontinuous or have any shape, mean, and/or variance.

These minimal assumptions make it equally likely that any measurement has any rank from 1 to $N$. Whatever the unknown underlying distribution is, the rank of measurement \#1 is a random selection from the integers 1 to $N$. The probability of any particular integer is $1 / N$. Measurement \#2 has equal probability of having any of the remaining $N-1$ ranks. (This key observation that the ranks are uniformly distributed random integers underlies many of these distribution-free statistical methods.)

Consider a set of $N$ balls, marked with the integers: $1-N$. The rank-sum is a statistic obtained by drawing $m$ of these balls, and adding their marked values. Ignoring for the moment that the drawing is without replacement, then each integer is a random number taken with equal probability $1 / N$ from the uniform discrete probability distribution with range 1 to $N$. Each one has $s$-expected value

$$
\mu=\frac{1}{N} \cdot \sum_{k=1}^{N} k=\frac{1}{2}(N+1) .
$$

The sum of $m s$-independently sampled integers strongly converges to a Gaussian distribution, and its mean is $m \cdot \mu$; thus $\mu(R)=(1 / 2) m \cdot(N+1)$, which is (A-1). Proving (A-2) is more difficult, due to negative covariance between pairs of ranks caused by the nonreplacement [17].

Rank-sum tests as used and described here should be distinguished from other rank-sum tests used for tests of location (mean) shift between 2 data sets, and tests of paired data [18].

\section{A.3 Choosing the Data Sample-Sizes}

For the Gaussian distribution approximation (A-4) to be valid, $m$ and $n$ have to be sufficiently large for the central limit theorem to be valid. The warning data set size $m=5$ used in this paper is too small for this purpose-and made worse by the many ties in this situation of counting discrete, rare errors: see Appendix A.5. Smaller $m$ values can detect failure sooner, after sudden attribute changes occur. Also, the experimental data sets included some drives with only 5 nonzero SMART samples. Equation (A-3) shows that the $R$ test statistical variability, $\mu / \sigma$, decreases to an asymptotic constant $\sqrt{1 /(3 \cdot m)}$ as $n$ is increased. Ample Good drive reference experimental data were available, and a somewhat arbitrary $n=50$ was chosen.

\section{A.4 Multivariate Rank-Sum Test}

An overall rank-sum for all $p$ attributes can be defined as

$$
R=\sum_{i=1}^{p} R_{i} .
$$

Because the attributes are defined to be monotonic (larger values mean increased failure risk, Section II-D), this multivariate rank-sum exploits any favorable $s$-correlations among the failure attributes, because they should be positive. Replacing the error counts by their ranks, automatically solves problems of scale and normalization. The individual attribute ranks can be simply added. Under the null hypothesis, the individual attribute measurements are assumed $s$-independent within each attribute (as in the single-variable rank-sum), and the attribute measurements are assumed $s$-independent of each other. For simplicity, assume that each of the attributes has the same warning and reference data set size, $m$ and $n$. The mean and variance of $R$ are then:

$$
\begin{aligned}
\mu[R] & =\sum_{i=1}^{p} \mu_{i}=p \cdot m \cdot \frac{1}{2}(N+1), \\
\sigma^{2}[R] & =\sum_{i=1}^{p} \sigma^{2}\left[R_{i}\right]=p \cdot n \cdot \frac{1}{12}(N+1) \cdot m .
\end{aligned}
$$

These values can be used along with $R$, as the $Q$-function $\operatorname{argu-}$ ment in (A-4).

\section{A.5 Data-Ties and Zeros}

The rank-sum test was originally developed for continuous data, with only accidental data-ties, but ties are prevalent in this case of discrete-valued SMART error count attributes. For example, most SMART attribute reads in Figs. 1-4 are tied 
at 0 . With the experimental data used here, best results were with 0 attribute-values ignored; this is not surprising because 0 error-counts give little information.

The standard recipe [17, section 5.1] for the rank-sum test, states that one imagines that tied variates are arbitrarily separated infinitesimally. Ranks are then assigned and the average rank of all infinitesimally close data is assigned to each of them. For example, if 10 identical error-counts of 1 occur in the reference plus warning data sets, then each of them gets rank $(1+10) / 2=5.5$ (because the zero error counts are ignored). This rule worked well with the discrete SMART data, and best preserves the rank-sum virtue that "drive failure trends producing simultaneous positive shifts in the attributes" will produce large changes in the rank-sum, toward the failure limit.

\section{A.6 Setting the Rank-Sum Failure Limit Parameter}

Equations (A-1)-(A-4) change and lose accuracy with ties, although the rank-sum remains a robust statistical test [17]. Discrete valued rare-error attributes can produce enough ties that the Gaussian approximation leading to (A-4) is inaccurate. As a rough rule, the number of untied values in the smaller data should exceed 20 [18]. For the experimental data here, the $s$-significance level had to be set numerically to get a desired FAR.

A good method to do this is simply to find the rank-sum limit producing the desired FAR in the won' $t-f a i l$ drives. Average over "all experimental drives" and "all warning sets of $m$ sequential attribute reads of each drive" (all possible SMART read times). This was tested on the new rank-sum tests (their ORed SMART flag result) and on the (single) multivariate rank-sum test.

Section II-E discusses why this should be a conservative estimate of production drive FAR.

\section{ACKNOWLEDGMENT}

The authors thank T. Nelson, C. Reynolds, and C. Camp of the Quantum Corporation for their assistance and for supplying the experimental data. They also thank W. H. Tran, R. Bohn, and S. Schultz.

\section{REFERENCES}

[1] E. Grochowski, "Future trends in hard disk drives," IEEE Trans. Magn., vol. 32, no. 3, pp. 1850-1854, May 1996.

[2] J. Yang and F-B Sun, "A comprehensive review of hard-disk drive reliability," in Proc. Annual Reliability \& Maintainability Symp., 1999, pp. 403-409.

[3] ATA SMART Feature Set Commands. Small Form Factors Committee SFF-8035. [Online]. Available: www.t13.org.

[4] J. Yang and X. K. Zunzanyika, "Field reliability projection during new (disk drive) product introduction," in Proc.-Annual Technical Meeting of the Inst. of Environmental Sciences, 1966, pp. 58-67.

[5] J. G. Elerath, "Specifying reliability in the disk drive industry," in Proc. Annual Reliability \& Maintainability Symp., 2000, pp. 194-199.

[6] A. S. R. Murty and V. N. A. Naikan, "Condition monitoring strategy-A risk based interval selection," in Int. J. Production Research: Taylor \& Francis, Jan. 1996, vol. 34, pp. 285-296.

[7] B. E. Preusser and G. L. Hadley, "Motor current signature analysis as a predictive maintenance tool," in Proc. American Power Conf., vol. 1, May 1991, pp. 286-291.

[8] D. R. Lewin, "Predictive maintenance using PCA," Control Engineering Practice, vol. 3, no. 3, pp. 415-421, Mar. 1995.

[9] "Drive industry endorses protocol for predicting disk drive failure," Data Storage Magazine, vol. 2, no. 9, Sept. 1995. [Online]. Available:http://ds.pennwellnet.com.
[10] SMART monitor software information, , www.seagate.com/support/kb/disc/smart.html, www.storage.ibm.com/hdd/support/download.htm, http://support/wdc.com/dlg/disclaim.asp.

[11] J. D. Coker, R. L. Galbraith, and G. J. Kerwin, "Magnetic characterization using elements of a PRML channel," IEEE Trans. Magn., pt. 1, vol. 27, no. 6, pp. 4544-4548, Nov. 1991.

[12] T. J. Chainer and E. J. Yarmchuk, "A technique for the measurement of track misregistration in disk file," IEEE Trans. Magn., pt. 2, vol. 27, no. 6, pp. 5304-5306, Nov. 1991

[13] B. C. Schardt, E. Schreck, and R. Sonnenfeld et al., "Flying height measurement while seeking in hard disk drives," IEEE Trans. Magn., pt. 1, vol. 34, no. 4, pp. 1765-1767, Jul. 1998.

[14] K. Rothman and S. Greenland, Modern Epidemiology, 2nd ed: Lippencott-Raven, 2000, p. 509.

[15] F. Wilcoxon, "Individual comparisons by ranking methods," Biometrica, vol. 1 , pp. 80-83, 1945

[16] P. D. Bridge and S. S. Sawilowky, "Increasing physicians' awareness of the impact of statistics on research outcomes: Comparative power of the t-test and Wilcoxon ran-sum test in small samples applied research," $J$. Clinical Epidemiology, vol. 52, no. 3, pp. 229-235, Mar. 1999.

[17] W. Conover, Practical Nonparametric Statistics, 3rd ed: Wiley, pp. 271-281.

[18] E. L. Lehman and H. J. M. D'Abrera, Nonparametrics-Statistical Methods Based on Ranks: Prentice-Hall, 1999.

[19] SCSI, "'Mode sense' code 'Failure prediction threshold exceeded'," American National Standards Institute, 11 W. 42nd St.; New York, NY 10036 , www.t10.org.

Gordon F. Hughes is the Associate Director of the Center for Magnetic Recording Research at the University of California, San Diego, and a Fellow of the IEEE. He is the principal investigator of the UCSD SMART project on disk drive predictive failure. He received his B.S. in physics and Ph.D. in electrical engineering from Cal Tech. Before 1983 he worked for Xerox PARC, on magnetic recording research for disk drives, then joined Seagate Technology as Senior Director of Recording Technology. At Seagate he worked on recording heads, disks, and systems, was part of the team that established sputtered thin film disk media as today's standard, and formed Seagate's first Material and Process Lab and Contamination Control Group for drive reliability analysis.

Joseph F. Murray received the B.S. (1998) in electrical engineering at the University of Oklahoma, and M.S. in electrical and computer engineering from the University of California, San Diego (UCSD). He is a Ph.D. student at UCSD, and his interests include pattern recognition, learning theory and computer vision.

Kenneth Kreutz-Delgado is a Senior Member IEEE; he received the M.S. in physics and Ph.D. in engineering systems science from the University of California at San Diego (UCSD) where he is a professor in the Department of Electrical and Computer Engineering. Prior to joining the Faculty of UCSD in 1989, Dr. Kreutz-Delgado was a researcher at the Jet Propulsion Laboratory, California Institute of Technology, where he worked on the development of intelligent space telerobotic systems. His current research interests include applications of statistical data analysis and learning theory, nonlinear signal processing, and computational intelligence to communication systems, bioinformatics, predictive failure analysis, machine intelligence, and robotics.

Charles Elkan is an associate professor in the Department of Computer Science and Engineering at the University of California, San Diego. His main research interests are in artificial intelligence and data mining. His research has led to 2 best-paper awards and first place in two international data-mining contests. In 1998-1999, Dr. Elkan was a visiting associate professor at Harvard. He earned his Ph.D. at Cornell University in computer science, and his B.A. at Cambridge University in mathematics. 